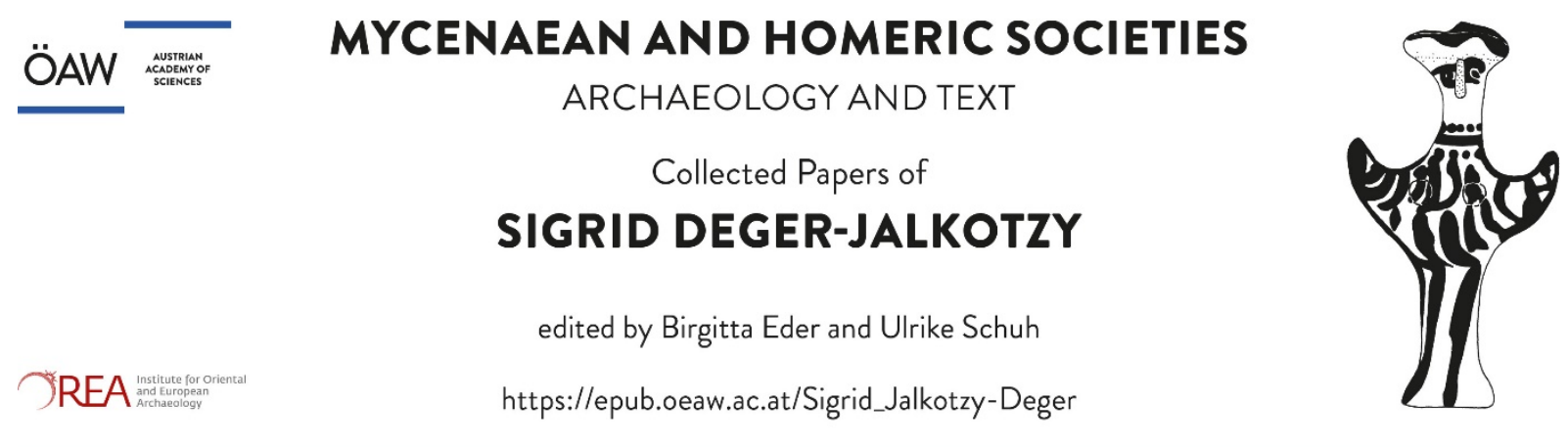

41.

S. Deger-Jalkotzy

\title{
Ionische Wanderung
}

in: Der Neue Pauly. Enzyklopädie der Antike 6 (Stuttgart - Weimar 1999) 648-651

(C) J.B. Metzler [Brill] https://referenceworks.brillonline.com/browse/brill-s-new-pauly mit freundlicher Genehmigung / with kind permission

Dieses Dokument darf ausschließlich für wissenschaftliche Zwecke genutzt werden (Lizenz CC BY-NC-ND), gewerbliche Nutzung wird urheberrechtlich verfolgt.

This document is for scientific use only (license CC BY-NC-ND), commercial use of copyrighted material will be prosecuted. 


\section{DER NEUE PAULY}

\section{(DNP)}

Fachgebietsherausgeber

Prof. Dr. Gerhard Binder, Bochum

Kulturgeschichte

Prof. Dr. Rudolf Brändle, Basel

Christentum

Prof. Dr. Hubert Cancik, Tübingen

Geschäftsführender Herausgeber

Prof. Dr. Walter Eder, Bochum

Alte Geschichte

Dr. Karl-Ludwig Elvers, Bochum

Alte Geschichte

Prof. Dr. Bernhard Forssman, Erlangen

Sprachwissenschaft; Rezeption: Sprachwissenschaft

Prof. Dr. Fritz Graf, Basel

Religion und Mythologie; Rezeption: Religion

PD Dr. Hans Christian Günther, Freiburg

Textwissenschaft

Prof. Dr. Berthold Hinz, Kassel

Rezeption: Kunst und Architektur

Dr. Christoph Höcker, Kissing

Klassische Archäologle (Architekturgeschichte)

Prof. Dr. Christian Hünemörder, Hamburg Naturvissenschaften und Technik; Rezeption:

Naturvissenschaften

Dr. Margarita Kranz, Berlin

Rezeption: Philosophie

Prof. Dr. André Laks, Lille

Philosophie

Prof. Dr. Manfred Landfester, Gießen

Geschäftsführender Herausgeber: Rezeptions- und

Wissenschaftsgeschichte; Rezeption: Wissen-

schafts- und Kulturgeschichte

Prof. Dr. Maria Moog-Grünewald, Tübingen

Rezeption: Komparatistik und Literatur

Prof. Dr. Dr. Glenn W. Most, Heidelberg

Griechische Philologie
Prof. Dr. Beat Näf, Zürich

Rezeption: Staatstheorie und Politik

PD Dr. Johannes Niehoff, Freiburg

Judentum, östliches Christentum, byzantinische Kultur

Prof. Dr. Hans Jörg Nissen, Berlin Orientalistik

Prof. Dr. Vivian Nutton, London Medizin; Rezeption: Medizin

Prof. Dr. Eckart Olshausen, Stuttgart

Historische Geographie

Prof. Dr. Filippo Ranieri, Saarbrücken

Rezeption: Rechtsgeschichte

Prof. Dr. Johannes Renger, Berlin

Orientalistik; Rezeption: Alter Orient

Prof. Dr. Volker Riedel, Jena

Rezeption: Erziehungswesen, Länder (II)

Prof. Dr. Jörg Rüpke, Potsdam

Lateinische Philologie, Rhetorik

Prof. Dr. Gottfried Schiemann, Tübingen

Recht

Prof. Dr. Helmuth Schneider, Kassel

Geschäftsführender Herausgeber; Sozialund Wirtschaftsgeschichte, Militärwesen; Wissenschaftsgeschichte

PD Dr. Christine Walde, Basel

Religion und Mythologie

Prof. Dr. Dietrich Willers, Bern

Klassische Archäologie

(Sachkultur und Kunstgeschichte)

Dr. Frieder Zaminer, Berlin

Musik; Rezeption: Musik

Prof. Dr. Bernhard Zimmermann, Fresburg Rezeption: Länder (I) 


\section{DER NEUE PAULY \\ Enzyklopädie der Antike}

Herausgegeben

von Hubert Cancik und

Helmuth Schneider
Altertum

Band 6 Iul-Lee

Verlag J. B. Metzler 


\section{Inhaltsverzeichnis}

Dic Dentsclic Bibliothek - CIP-Einlıcitsaufnalume

Hinweise für die Benutzung

Der neue Pauly' : Enzyklopädie der Antike/hrsg. von Hubert Cancik und Helmuth Schneider. Stuttgart ; Weimar : Metzler, 1999

ISBN 3-476-0I 476-2

NE: Cancik, Hubert [Hrsg.]

Bd. 6. Iul-Lee - 1999

ISBN 3-476-0I 476-2
Karten- und Abbildungsverzeichnis

Verzeichnis der Autoren

IX

Verzeichnis der Übersetzer

XII

Mitarbeiter in den FG-Redaktionen

XII

Lemmata

I

Corrigenda zu Band 3 bis 5

Gedruckt auf chlorfrei gebleichtem, säurefreiem und alterungsbeständigem Papier

Dieses Werk einschließlich aller seiner Teile ist urheberrechtlich geschützt. Jede Verwertung außerhalb der engen Grenzen des Urheberrechtsgesetzes ist ohne Zustimmung des Verlages unzulässig und strafbar. Das gilt insbesondere für Vervielfältigungen, Übersetzungen, Mikroverfilmungen und die Einspeicherung und Verarbeitung in elektronischen Systemen.

ISBN 3-476-0I 470-3 (Gesamtwerk)

ISBN 3-476-0I 476-2 (Band 6 lul-Lee)

(C) 1999 J.B. Metzlersche Verlagsbuchhandlung und Carl Emst Poeschel Verlag $\mathrm{GmbH}$ in Stuttgart

Typographie und Ausstattung: Brigitte und Hans Peter Willberg Grafik und Typographie der Karten: Richard Szydlak

Abbildungen: Günter Müller Satz: pagina $\mathrm{GmbH}$, Tübingen Gesamtfertigung: Franz Spiegel Buch GmbH, Ulm

Printed in Germany

Verlag J. B. Metzler Stuttgart · Weimar

\section{Redaktion}

Jochen Derlien Dr. Brigitte Egger

Susanne Fischer Dietrich Frauer Mareile Haase Dr. Ingrid Hitzl Heike Kunz Vera Sauer Christiane Schmidt Dorothea Sigel Anne-Maria Wittke 
Die umfangreichste und in ihrer historischen Wirkung bedeutendste Welle ist die sog. "große griech. K.", die von zahlreichen griech. Gemeinden (außer Athen) ausgeht und in der Blütezeit zw. ca. 750 bis $580 \mathrm{v}$. Chr. wohl zur Verdoppelung der griech. Stadtstaaten im Mittelmeer führt. Schwerpunkte sind das südl. Italien $(\rightarrow$ Magna Graecia), Sizilien, die nördl. Ägäis und das Schwarzmeergebiet (s.u. IV.). Seit dem 6. Jh. verflechten sich die K.-Bewegungen im westl. Mittelmeer und es kommt zunehmend zu Konflikten mit den Karthagern in Sizilien und Spanien und/oder den Etruskern in Nord- und Mittel-It., wohin diese sich seit dem 9. Jh. und verstärkt ab dem 7. Jh. ausgedehnt haben (s.u. V.). Die etr. K. endet im Süden mit der Seeschlacht gegen sizil. Griechen bei $\rightarrow$ Kyme [2] (474 v. Chr.) und im Norden mit dem Eindringen der Kelten. Deren Wanderzüge im 4. und 3. Jh. nach It., Südosteuropa und Anatolien sind histor. belegt, aber arch. schwer faßbar und kaum als K.-Unternehmen zu werten ( $\rightarrow$ Keltische Archäologie).

Im 5. Jh. vollzieht sich ein Wandel im Bild der K.: Hatten bisher regelmäßig Handelsinteressen, wirtschaftliche Not oder polit. Probleme zur Gründung von Kolonien geführt, so dient die Ansiedlung von Kolonisten nun primär dazu, Herrschaft in ausgedehnten Räumen zu sichern (nicht zu gewinnen!). Dies wird bereits im Seereich Athens sichtbar $(\rightarrow$ Attisch-Delischer Seebund; $\rightarrow$ Kleruchoi [1]); völlig deutlich wird der Zweck der mil. Sicherung und der Förderung der Reichsverwaltung in den zahlreichen Kolonien Alexandros' [4] d. Gr. und der hell. Könige (s.u. VI.). Die röm. K. ist von Beginn an (seit dem 4. Jh. v. Chr.) auf diese Ziele gerichtet und übernimmt erst seit dem E. des 2. Jh. v. Chr. zusätzlich die Aufgabe der Versorgung armer Bevölkerungsschichten und von Veteranen $\rightarrow$ coloniac). Während jedoch die Kolonien in den hell. Reichen wenig zur Hellenisierung der Unterworfenen beitragen, fördern die röm. Kolonien v.a. in den Provinzen wirksam die Romanisation der Reichsbevölkerung ( $\rightarrow$ Romanisierung; Romanisation).

W. EI)

Karten-Lir.: B. D'Agostino, Relations between

Campana, Southern Etruria, and the Aegean in the Eighth

Century B.C., in: J.-P. Descoeudres (Hrsg.), Greek

Colonists and Native Population, 1990, 73-85 - F. M.

Andraschко, K. Sснмidt, Orientalen und Griechen in

Ägypten. Ausgrabungen auf Elephantine, in: FS H.G.

Niemeyer, 1998, 46-67 P. Barceló, Die Phokäer im

Westen, in: FS H.G. Niemeyer, 1998, 605-614 • J.-P.

Descoeudres (Hrsg.), Greek Colonists and Native

Population, $19900^{\circ}$ H. MAtтHÄUs, Zypern und das

Mittelmeergebiet - Kontakthonzonte des spiten 2. und

frühen I. Jt. v.Chr., in: FS H. G. Niemeyer, 1998, 73-91 *

H. G. Niemeyer, The Phoenicians in the Mediterranean: A

Non-Greek Model for Expansion and Settlement in

Antiquity, m: J.-P. Descoeudres (Hrsg.), Greek Colonists and Native Population, I990, $460-489^{\circ}$ H. G. Nicmeyer (Hrsg.), Phônizier im Westen (Madrider Beıtràge 8), i982 * G. R. Tsetskhladze, F. DE Angelis (Hrsg.), The

Archaeology of Greek Colonisation. Essays Dedicated to Sir John Boardman, 1994.

\section{IONISCHE WANDERUNG}

A. Allgemein B. Historizität

C. Chronologie D. Herkunft der

IONIER AUS ATtiKa UND ATHEN

E. Herkunft aus anderen griech. Regionen

\section{A. Allgemein}

Der mod. Begriff "Ionische Wanderung" (I. W.) bezeichnet die nach ant. Tradition von Athen ausgehende und von den Söhnen des $\rightarrow$ Kodros organisierte und geleitete Auswanderung festländischer Griechen nach Kleinasien (zusammenfassend Hdt. I, I45-147; Strab. I 4, I,3; Paus. 7,2,I-4); er wurde aufgrund der großen Zahl und der Herkunft der Teilnehmer aus zahlreichen Regionen Griechenlands geprägt (Pylier: Mimnermos fr. 9 West; Athener mit Herkunft aus Pylos oder Messenien wie im Fall der Kodriden: Hellanikos FGrH $4 \mathrm{~F}$ 125; Paus. 7,2,3; Ionier aus Achaia: Hdt. I, 145 f.; 7,9495, I ; Strab. 8,7,I; Paus. 7,1,1-6; Teilnehmer aus anderen griech. Regionen: Hdt. I, 146 ; umfassende Auswertung ion. Lokaltraditionen, Institutionen, Monats-, Personen- und Ortsnamen, Kulte und Feste bei [16. Teil I]). In den Quellen wird das Unternehmen als (Ioniké) $\rightarrow$ apoikía bezeichnet und auch im Stil einer Koloniegründung beschrieben. Als Ursache der I. W. gilt der Streit um die Königswürde in Athen unter den Söhnen des Kodros, den Medon gegenüber Neleus und weiteren Brüdern gewann (Hellanikos FGrH $4 \mathrm{~F} 125$; Paus. 7,2, I ; Ail. var. 8,5). Die Zeit der I. W. scheinen die Quellen in der 4. Generation nach dem Fall Troias bzw. zwei Generationen nach der Rückkehr der $\rightarrow$ Herakleidai anzusetzen (relativ-chronolog. Analyse bei [16. 307-324; 14. 326-330]). Auf der Basis hell. Chronologien wurde für diese relative Abfolge ein absolutes Datum in 11.Jh. v.Chr. errechnet ([16], danach [20. 392-395]).

Der histor. Gehalt der vielfach fragmentarisch, oft widersprüchlichen und keinesfalls homogenen Aussagen der Quellen zur I. W. (zusammengestellt bei [16]) wird in der mod. Forsch. sehr unterschiedlich beurteilt. Im Zentrum der Debatte stehen Historizität, Chronologie und die Beteiligung Athens bzw. anderer griech. Regionen.

\section{B. Historizität}

Die Besiedlung der ägäischen Küstenregion Kleinasiens durch einwandernde Griechen aus dem Mutterland wird heute allg. als histor. Faktum akzeptiert. Die Erkenntnisse der mod. Dialektforschung zur Verbreitung und Entwicklung der ion. Dialektgruppe lassen keine andere Erklärung zu (vgl. [19. 96-103; 124-133]; $\rightarrow$ Ionisch).

C. Chronologie

Der heutige Stand der arch. Forsch. für Kykladen, Westkleinasien und die vorgelagerten Inseln [17. 329$344 ; 20.166-170]$ unterstützt weiterhin die Datier. der griech. Landnahme in Ionien in submyk. und protogeom. Zeit $[5.785-790 ; 17 ; 18]$, d.h. in das I 1. Jh. v. Chr., kommt also den ant. Berechnungen er- 
staunlich nahe. Ebenfalls im I I. Jh. (E. von SH III C Spät und Submyk.) läßt sich in den meisten Landschaften der Peloponnes ein weitgehender Siedlungsrückgang beobachten (s.u. E.). Die myk. Funde in Westkleinasien [I s; 9; 6; I2; I I] wurden dagegen in ihrer Bed. für die I. W überschätzt (etwa von [3; I6; 20]. Myk. Gefäße, Vasenscherben und einzelne andere Objekte im nachmaligen Ionien bezeugen zwar Kontakte mit der myk. Welt, aber nicht notwendig die Präsenz myk. Siedler. Selbst in Ephesos können myk. Funde des früheren I4. Jh. v. Chr. aus einem (stark gestörten) Grab und die Zeugnisse myk. Kultübung beim Artemision [1. 27f.] den myk.-griech. Charakter der spätbrz. Siedlung nicht nachweisen, da sie auch von Einzelpersonen oder Gruppen myk. Kulturprägung, etwa Bewohnern einer Handelskolonie oder eines Stützpunkts für den Seehandel inmitten eines sonst rein anatolischen Milieus stammen können. Ähnliches gilt für das (h. verschwundene) "Kuppelgrab" von Kolophon. Neben den arch. Quellen haben neuere hethitische Textfunde es unwahrscheinlich gemacht, in Westkleinasien $\rightarrow$ Achijawa oder einen anderen griech. Siedlungsraum z.Z. des hethit. Großreiches zu lokalisieren (vgl. [7. 2 I7-22 I; 2. Karte 3]).

Anders war die Situation in SW-Anatolien, wo Milet, Iasos [s] und Müsgebi als rein myk. Siedlungen des I4. und I 3 . Jh. gelten müssen. In $\rightarrow$ Miletos (wohl auch Iasos) hatten sich zuvor schon minoische Kolonisten angesiedelt (zuletzt [12]). Die Identität von Milet mit der Stadt Millawanda, die nach hethit. Texten (zumindest zeitweise) unter dem Einfluß von Achijawa stand [8], ist nun auch aus arch. Sicht sehr wahrscheinlich [12]. Kontakte dieser Region mit dem myk. Festland spiegeln sich wohl in den Ethnika mi-ra-ti-ja, ki-ni-di-ja, a- ${ }^{*} 6_{4}-j a$ (= wahrscheinlich $a$-si-wi-ja) der $\rightarrow$ Linear B-Texte von Pylos, die sich auf Frauen aus Milet, Knidos und *'A $\sigma$ Fí $\alpha$ (Aswía) = 'A ${ }^{\prime} \alpha$ (Asía) beziehen, deren niedriger Status die Herkunft über Sklavenhandel nahelegt. In Samos enden myk. Funde mit dem I3. Jh. In Chios wurde die bedeutende Siedlung des I2. Jh. von Emporio in SH III im C Spät zerstört.

Insgesamt eignen sich die westkleinasiat. Zeugnisse für Kontakte mit dem myk. Griechenland ebensowenig für den Nachweis einer Hellenisierung Westkleinasiens in myk. Zeit wie die myk. Siedlungen SW-Anatoliens. Sie zeigen aber, daß die Kolonisation Ioniens in einem den Griechen bereits bekannten Gebiet erfolgte.

\section{Herkunft der IONIER aUS}

\section{Attika und Athen}

Die Herkunft der Ionier aus Attika und Athen wird in der mod. Forsch. meist als histor. akzeptiert (Überblick bei [14. 336]). Als wichtigste Argumente gelten gemeinsame Phylennamen bei Athenern und Ioniern, der gemeinsame Brauch des Apaturienfestes ( $\rightarrow$ apatúria) und die fast übereinstimmende Aussage der lit. Quellen (Hdt. 5,97,2; 7,94-95,I; 9,106,3 u.ö.; Thuk. I,2,6; $\mathrm{I}, \mathrm{I} 2,4)$. Doch werden diese Zeugnisse aus unterschiedlichen Gründen auch als Ergebnis von Erfindungen oder doch Manipulationen älterer Überl. im Interesse der att. Politik gesehen ([3; I6; I4; 20,367-404]; dagegen zuletzt wieder vehement [I8]). Für eine frühe Verbindung von Festland und Ioniern spricht auch, daß sich bei Homer (Hom. Il. 13,685) und Solon (fr. 4 D) im Ethnikon Iáones und im Landesnamen Iaonía die ältere, auf *'lóffoves (*Iáwones) zurückgehende Form des Ioniernamens zeigt. Sie ist in den Linear B-Texten von Knossos durch $i$-ja-wo-ne belegt und (in KN B I64,4) als Ethnikon /Iawones/ interpretierbar. Zudem leiten sich die vorderasiat. Bezeichnungen für die Griechen als $\mathrm{Ge}-$ samtheit von *'Iófoves ab (hebr. jawan, äg. jwn(n), pers. yauna), müssen also noch vor dem Schwund des Digamma $(F)$ gebildet oder aus einem nicht-ion. Dialekt entlehnt worden sein. Die Herleitung von Íōnes aus *Iáwones ist dagegen nicht zwingend, da auch eine mit dem eponymen Heros Íōn assoziierte Neubildung vorliegen kann [4].

E. Herkunft aus anderen griech. Regionen

Der oben erwähnte Siedlungsrückgang in der Peloponnes am E. der myk. Periode zeigt sich in Achaia und Messenien bes. radikal (vgl. [20. Karte 3]). In Athen dagegen läßt der arch. Befund auf Siedlungszuwachs ab der ausgehenden myk. und submyk. Periode schließen [1 3; 20. I I 5-I I 7 und Fig. 3; 21. 60-75], so daß die ant. Berichte über die Herkunft der Ionier aus Messenien und Achaia an Glaubwürdigkeit gewinnen. V.a. für Messenien verstärkten die Entdeckung des nach den Linear B-Texten eindeutig als puro/Pylos/identifizierten Palastes von Ano Englianos ( $\rightarrow$ Pylos), seine Zerstörung am E. des I3. Jh. und der folgende rapide Siedlungsrückgang die Anerkennung eines gewissen histor. Gehalts der ant. Trad. über die zumindest teilweise Herkunft der Ionier aus Pylos. Dabei wird der in den meisten Quellen überlieferte Weg über Athen - Aufnahme pylischer Flüchtlinge, Übernahme des Königtums in Athen durch das pylische Königshaus der Neleiden mit $\rightarrow$ Kodros als glänzendstem Vertreter, Auswanderung der Pylier nach Kleinasien unter dem Kodros-Sohn $\rightarrow$ Neleus (Stammbaum bei Hellanikos FGrH 4 F I25) -, aber auch die bei Mimnermos (fr. 9 WEST) erwähnte direkte Wanderung von Pylos nach Ionien in Betracht gezogen (vgl. [I 8. 3 I I]). Für Boiotien und Thessalien reicht dagegen der derzeitige Stand der arch. Forsch. nicht aus, um eine Herkunft der Ionier aus Mittelgriechenland zu stützen (so [I6]; vorsichtige Zustimmung bei [I8. 30I]).

$\rightarrow$ Ionisch; Iones

1 A. Bammer, U. Muss, Das Artemision von Ephesos, 1996 2 T. Bryce, The Kingdom of the Hittites, 1998

3 F. Cassola, La Ionia nel mondo miceneo, I957

4 J. Chadwick, The Ionian Name, in: K. H. KinZl (Hrsg.), Greece and the Mediterranean in Ancient History and Prehistory. Studies ... Fritz Schachermeyr, 19775 J. N. Соок, Greek Settlements in the Eastern Aegean and Asia Minor, in: $\mathrm{CAH} \mathrm{II}{ }^{3} 2,1975,773-8046$ E. B. FreNCH, Turkey and the East Aegean, in: C. Zerner (Hrsg.), Wace and Blegen, I993, I 55-I 587 O.R. GuRney, Hittite Geography, in: H. Otten u.a. (Hrsg.), Hittite and Other 
Anatolian and Near Eastern Studies in Honour of Sedat Alp, I992, 21 3-221 8 S. Heinhold-Krahmer, s. v. Milawa(n)da, in: RLA 8, 188f. 9 CH. MEE, Aegean Trade and Settlement in Anatolia in the Second Millenium B. C., in: AS 28, 1978, 121-15610 W. Müller-WIener (Hrsg.), Milet 1899-1980, 198611 W.-D. Niemeier, The Mycenaeans in Westerm Anatolia and the Problem of the Origins of the Sea Peoples, in: S. Girin u.a. (Hrsg.), Mediterranean Peoples in Transition: Thirteenth to Early Tenth Centuries B. C., 1998, 17-65 12 B. Niemeier, W.-D. Niemeier, Milet 1994-1995,

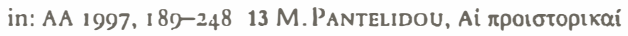

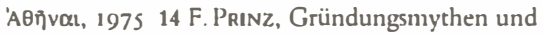
Sagenchronologie, 1979, 314-376 15 L. RE, Presenze nucenee in Anatolia, in: M. Marazzi u.a. (Hrsg.), Traffici nucenei nel Mediterraneo, 1986, 343-358

16 M. Sakellariou, La migration grecque en Ionie, 1958 17 F. SChachermeyr, Die Ägäische Frühzeit 4, 1980 18 Ders., Die griech. Rückerinnerung im Lichte neuer Forschungen, 1983, 296 320 19 R. Sснм ITT, Einführung in die griech. Dialekte, 1977, 96-103: 124-133 $20 \mathrm{~J}$. VANSChOONWINKel, L'Égée et la Méditerranée onentale à la fin du deuxième millénaire, $199121 \mathrm{~K} . \mathrm{W}$. Welwel, Athen, 1992.

\section{Phönizische Kolonisation}

Die phöniz. Expansion in den Westen am Ende des 2. Jt.v. Chr. gründet in den traditionellen Handelsbeziehungen der spätbrz. Koinc der Levante, Zyperns und Anatoliens mit der Ägäis und dem zentralen und westl. Mittelmeerraum. Nach dem Ausgang der Krise des 12. Jh. ( $\rightarrow$ "Seevölkerwanderung"), die sich für die phöniz. Stadtstaaten der Levanteküste - hier lit. oder arch. kaum überl. - in Dauer und Intensität unterschiedlich ausgewirkt hatte, die jedoch offenbar meist glimpflich verlaufen war, zielte die phöniz. Expansion auf die reichen Silber-, Kupfer- bzw. Zinnerzvorkommen Zyperns $(\rightarrow$ Kypros), Etruriens $(\rightarrow$ Etrusci, Etruria), $\rightarrow$ Sardiniens und der Iberischen Halbinsel $(\rightarrow \mathrm{Py}-$ renäenhalbinsel). Sie diente der Versorgung der hochspezialisierten phöniz. Künstler und Handwerker sowie dem profitablen Zwischenhandel (u.a. mit Assyrien, auch Tributleistungen). Rasch und sukzessive eroberten sich die Phönizier die Märkte des 2. Jt. im zentralen und westl. Mittelmeerraum zurück. Wirtschaftlich erstarkt, sollten $\rightarrow$ Byblos, $\rightarrow$ Sidon und $\rightarrow$ Tyros als die bedeutendsten phöniz. Metropolen alsbald wieder das polit. Geschehen im Phönizien der frühen Eisenzeit bestimmen.

Schon früh müssen die phöniz. Handelsverbindungen, etwa mit Ägypten, intensiv und stabil gewesen sein, wie der Ber. des Wen-Amun (ca. 1075 v. Chr.) glauben macht: 50 Schiffe der sidonischen und 20 der byblischen Handelsflotte standen im Warenaustausch mit Ägypten. Nicht von der Hand zu weisen, doch hinsichtlich der v.a. im Westen noch fehlenden arch. Befunde mit Bedacht zu nutzen, sind jene schriftlichen Nachr., die uns die griech. und röm. Geschichtsschreibung über den Gründungszeitpunkt der frühesten phöniz. Städte bzw. Niederlassungen vernitteln: Gründung von Tyros I 198/1 18 s v. Chr. (Ios. ant. Iud. 8,62 bzw. Iust. 18,3,5), von $\rightarrow$ Kition Anf. 12. Jh. (Verg. Aen. 1,619-626), von $\rightarrow$ Utica I IOI (Aristot. mir. 134; Plin. nat. 16,216), von $\rightarrow$ Gades I I04/3 (Vell. I, 2, I-3) und von $\rightarrow$ Lixus (Plin. nat. 19, 63). Myth. Heldentaten, wie die Gründung von $\rightarrow$ Salamis/Kypros durch Teukros, das Dahinscheiden des Herakles und die Rückkehr der Herakliden $(\rightarrow$ Herakleidai), der Fall der athenischen Monarchie oder der Untergang von Troia (I I 84 v. Chr.) galten offenbar als sichere chronologische Eckpunkte der phöniz. Expansion im Mittelmeerraum, denen zufolge sie am Beginn des 12. Jh. ihren Anfang nahm.

Treibende Kraft hinter der phöniz. Expansion waren relativ kleine, ebenso mächtige und selbständige wie vom $\rightarrow$ Handel (II. Phönizien) existentiell abhängige phöniz. Stadtstaaten. Diese Expansion, deren polit.wirtschaftl.-organisatorische Struktur und Hintergründe sich in den darauffolgenden rund 500 Jahren kaum verändert haben, schlug sich in zwei teils aufeinanderfolgenden, teils sich überlagernden arch. Phänomenen nieder. Gut belegt ist von Anf. an die Allgegenwart phöniz. "merchant venturers" und Handwerker bzw. ihrer Produkte - zum überwiegenden Teil Luxus- und Prestigeimporte, aber auch (zunächst nur im östl. Mittelmeerraum) die ebenso schlichte wie offenbar attraktive Keramik - in den reich ausgestatteten Adelsgräbern Zyperns (Palaiopaphos-Skales, Salamis) und der Ägäis (Rhodos, Kos, Samos, Kreta, Euboia, Athen, Korinth). Nach und nach entstanden phöniz. Handelskontore und Werkstätten (cnoikismoí) in den kypr. und griech. Siedlungen, die sich sowohl die Rohstoffquellen und den lokalen Markt zunutze machten, als auch in engem und dauerhaftem Kontakt mit der Levante standen.

Für die weitere Expansion während des 10.-ๆ. Jh. nach Westen, in den zentralen Mittelmeerraum, nach It., Sardinien, die Atlantikküste Marokkos und schließlich auf die Iberische Halbinsel, sind die arch. Quellen noch sporadisch und bestätigen offenbar das Bild eines eher unregelmäßig, aber nicht notwendigerweise selten praktizierten phöniz. Fernhandels, dessen Wurzeln bis in die Brz. zurückreichen. Hier ist v.a. die biblische Überl. der von den Königen $\rightarrow$ Hiram I. (Tyros) und Salomo (Jerusalem) alle drei Jahre unternommenen Tarschisch-Fahrten zu nennen, die vern. dem sagenhaften Reich $\rightarrow$ Tartessos in Südspanien, dem Hinterland der tyrischen Kolonie Gadir galten ( $1 \mathrm{Kg}$ 10,22: 969-930 v. Chr.; Ez 27,12: 586 v. Chr.), eine Identifizierung, die plausibel, aber umstritten ist.

In einer zweiten Phase (8.- Mitte 7. Jh.v. Chr.) entstanden in diesen Ländern zahlreiche feste und nun auch arch. meist bis in das 6 . Jh. nachweisbare phöniz. Niederlassungen (Emporia), Faktoreien und Heiligtümer, vorwiegend an der Peripherie, mit Werkstätten und $\mathrm{Zu}$ gang zum Meer, teils in unmittelbarer Nähe zu den Wirtschaftsgebieten. In der Regel war das Hinterland nicht polit.-administrativ untergeordnetes oder gar unterworfenes Territorium; ökonomisch und technologisch waren die phöniz. Partner teils überlegen, teils angewiesen auf die Kooperation mit der indigenen Ari- 